\title{
Grouping Tolerant Level of Cocoa Genotypes Against Pod Rot Disease in Malaysia
}

\author{
Albert Ling Sheng Chang $^{1 *}$, Suhaida Salleh ${ }^{2}$, Nuraziawati Mat Yazik ${ }^{3)}$, Haya Ramba ${ }^{1}$, \\ and Ahmad Kamil Mohd Jaaffar ${ }^{1)}$ \\ ${ }^{1)}$ Malaysian Cocoa Board, 5-7 $7^{\text {th }}$ Floor, Wisma SEDCO, Lorong Plaza Wawasan, Off Coastal Highway, \\ Locked Bag 211, 88999 Kota Kinabalu, Sabah, Malaysia. \\ ${ }^{2)}$ Cocoa Research \& Development Centre Jengka, Malaysian Cocoa Board, Jalan Jengka 23, \\ P.O. Box 34, 28000 Temerloh, Pahang, Malaysia. \\ ${ }^{3)}$ Cocoa Research \& Development Centre Bagan Datuk, Malaysian Cocoa Board, \\ P.O. Box 30, Sg. Dulang Road, 36307 Sg. Sumun, Perak, Malaysia. \\ Corresponding author: albert@koko.gov.my \\ Received : 28 May 2020 / Accepted : 18 June 2020
}

\begin{abstract}
Cocoa pod rot disease (CPRD), caused by Phytophthora palmivora, is the main disease that caused major losses in Malaysia. It is important for screening the cocoa genotypes available in Malaysia for their tolerance level against the CPRD. This paper has an objective to select the potential genotypes tolerant to $P$. palmivora by grouping the cocoa genotypes available in Malaysia based on four tolerant levels such as tolerant, moderately tolerant, moderately susceptible and susceptible. The experiment was carried out at the laboratory of Plant Pathology at the Cocoa Development and Research Centre Jengka, Pahang using the detached pod test. Isolate of $P$. palmivora was obtained from a naturally infected cocoa pod in cocoa field at the Cocoa Research and Development Centre Tawau, Sabah, Malaysia then inoculated by a single point on the ridges of pod to 40 mature unripe pods of each tested genotypes. Fifty genotypes were tested in this study. The assessed disease severity was the rate of lesion area development from 1 to 7 days after inoculation and the proportion of pod area infected by CPRD. The disease severity was significantly different among tested genotypes showing tolerance variability against CPRD. Four nonlinear models consisted of Monomolecular model, Exponential model, Logistic model and Gompertz model were used to fit the proportion pod infection area curve. The best fitted Gompertz model was used in calculated the area under disease progress curve (AUDPC). The variability of both disease severity variables was used to group the genotypes into four tolerant levels using the $k$-means clustering method with 10 genotypes in group I (torelant), 14 genotypes in group II (moderately tolerant), 13 genotypes in group III (moderately susceptible) and 13 genotypes in group IV (susceptible). Six genotypes in group I, namely MCBC 13, PBC 221, BAL 209, KKM 19, QH 1176 and KKM 22 were identified to have lower disease severity values compared to control tolerant genotype PBC 123 that could be suggested to the farmers to be planted in the field.
\end{abstract}

Keywords: AUDPC, nonlinear model, cocoa genotypes, cocoa pod rot disease, tolerant level 


\section{INTRODUCTION}

Cocoa pod rot disease (CPRD), caused by Phytophthora palmivora, is the main disease that caused major losses in Malaysia. The symptom of the disease began with pod lesions as small, hard, dark spots on any part of the pod, at any stage of pod development (Lee et al., 2014). There are several control measures have been recommended against the CPRD such as agronomic practice and chemical control (Lee et al., 2013). Alternative to the chemical control on the CPRD was to use the biological agents as biofungicide (Pratama et al., 2013; Alsultan et al., 2019). Another approach to control the disease in long term is by developing the genetic resistance of cocoa tree against the CPRD in breeding program (Susilo \& Anita-Sari, 2014).

It is important to establish the effective screening method for selecting the tolerant genotypes in breeding program. However, selection of tolerant plant in the field would take many years as need to wait until a tree bears pods and the infection rates are not always sufficient under natural infection conditions may disrupt the reliability of assessment results (Cilas \& Despréaux, 2004). Therefore, understanding the disease severity progress from the lesion growth in the laboratory is more effective in assisting the breeder in identify the tolerance level of cocoa genotypes available, especially in Malaysia. This could be done by modeling the disease severity of its lesion growth over the time. The nonlinear models are commonly used to model temporal changes in the proportion of plant disease (Machiavelli, 2013). Four nonlinear models that commonly used to describe the disease progress curve are the exponential model, monomolecular model, logistic model and Gompertz model (Ling et al., 2017; Nayak et al., 2018). The work of characterizing the disease progress curve with nonlinear models and estimating the area under the disease progress curve (AUDPC) to related the estimated AUDPC to changes in the components of quantitative resistance has been carried out in many other plant diseases such as rice blast disease (Mukherjee et al., 2010), alternaria blight disease in Indian mustard (Brassica juncea) (Meena et al., 2011) and leaf blight disease in maize (Ohunakin et al., 2019). As in cocoa, the AUDPC was used in quantifying the effect of endophytic fungi against the CPRD in Indonesia (Tondok et al., 2012) but rarely used in cocoa screening test. Previous study has been carried out to develop the effective screening method on cocoa genotypes against CPRD using the detached pod test in laboratory and fitting nonlinear model on the disease severity based on the proportion of lesion size to the pod surface area followed by calculated the AUDPC to rank the tolerance level (Ling et al., 2017; Ling et al., 2018). The study highlighted that the Gompertz model was well-fitted to the disease severity and calculated AUDPC values for four genotypes of different level of resistance categories against CPRD gave high accuracy of the resistant categories ranking similar to the data published by Haya et al. (2012).

There are almost 50 cocoa commercial genotypes planted by farmers in Malaysia that may varied in terms of tolerance level against the CPRD (Lee et al., 2013). It is important to identify the tolerance level of these genotypes against the CPRD as its incidence in Malaysia was high due to the conducive environment for CPRD to attack cocoa pods (Tey, 1983). Therefore, it is critical to carry out the developed screening method (Ling et al., 2017) on these commercial genotypes available in Malaysia to identify the group of tolerance level of each genotype. The grouping method depends on the number of categories of tolerance level against 
CPRD. Janani \& Kumar (2014) has used four categories of resistance level (resistant, moderately resistant, moderately susceptible, and susceptible) to group its cocoa genotypes based on the disease severity described as percent pod area infected by CPRD at seven days after inoculation. The resistance category was defined as less than $25 \%$ area infected, moderately resistant was defined as $25-50 \%$ pod area infested, moderately susceptible was defined as $51-75 \%$ pod area infested and susceptible was defined as above $76 \%$ pod area infested. However, the resistance categories did not account the progress of the percentage of pod area infected from 1 to 7 days after inoculation. Meanwhile, Susilo \& Anita-Sari (2014) has categorised the cocoa genotypes based on five different resistance group, i.e. resistant, moderately resistant, moderately susceptible, susceptible and highly susceptible using the $k$-means clustering method based on the disease severity of lesion size up to $7^{\text {th }}$ day after inoculation of $P$. palmivora and rate of lesion enlargement. This $k$-means clustering method also known as disjoint clustering for large data sets was widely used methods compared to other clustering method due to its simplicity, ease of implementation and efficiency (Jain, 2010).

Thus, this paper is aimed to group the tolerance level of 50 available cocoa genotypes in Malaysia against the CPRD using the developed screening method (Ling et al., 2017) that involved i) estimate the disease severity; ii) fit the disease severity to the nonlinear models; iii) estimate the AUDPC of the best nonlinear model developed in each genotype and iv) group the cocoa genotypes based on tolerance level against the CPRD based on $k$-means clustering method. This information is useful for the breeders to be used as guideline in preparing the planting materials to be recommended for the farmers for planting.

\section{MATERIALS AND METHODS}

\section{Samples preparation}

Fifty cocoa genotypes that were widely planted by cocoa farmers in Malaysia were used in this study. The genotypes were divided into eight groups consisted of BAL (209 and 244), BR 25, DESA 1, KKM (1, $2,3,4,5,6,15,17,19,22,25,26,27$ and 28), $\operatorname{MCBC}(1,2,3,4,5,6,7,8,9$, $10,11,12,13$ and 14$), \operatorname{PBC}(112,123,130$, $131,137,139,140,159,179$ and 221), $\mathrm{QH}$ $(22,37,326,441,968,1003$ and 1176) and RP 1 . The pod samples were collected from farmers and the Cocoa Research and Development Centers in Peninsular Malaysia. Then brought back to Plant Pathology Laboratory in the Cocoa Research and Development Jengka, Pahang for the screening purpose against $P$. palmivora. In this study, genotypes of PBC 123 and KKM 4 which respectively categorized as tolerant and susceptible to P. palmivora based on Haya et al. (2012) and recommendation from Malaysian Cocoa Board plant pathologist were used as control.

The detached pod test described in Nyadanu et al. (2012) was used in preparing the pod sample for study the disease severity progress in each genotypes. The isolate of $P$. palmivora used in this study was obtained from a naturally infected cocoa pod in cocoa field at Cocoa Research and Development Centre Tawau, Sabah, Malaysia. In order to confirm the pathogen, macro and micro-morphological identification was done based on criteria explained by Drenth $\&$ Sendall (2004). The mature unripe pods (4-5 months old) were inoculated by a single point on the ridges of pod. 40 inoculated pods per genotype with $8 \mathrm{~mm}$ of mycelial plugs from seven days old $P$. palmivora culture grown on corn meal agar (CMA) were incubated at room temperature $\left(25 \pm 2^{\circ} \mathrm{C}\right)$ in the laboratory. The diameter of the established 
lesions were measured with a caliper meter throughout 1 to 7 days after inoculation (DAI).

\section{Measuring disease severity}

Disease severity in the study was measured with the rate of lesion area development from 1 to 7 days after inoculation as described in Susilo \& Anita-Sari (2014) and the proportion pod area infected by CPRD as described in Ling et al. (2017). In cocoa, it is important to measure the disease severity as the proportion pod area infected compared to the lesion size developed. The pod size has indirectly affected disease susceptibility because the pod size influences pod wetness duration due to dew and created chances of $P$. palmivora pathogen to infect the pod (Ten Hoopen et al., 2012). The proportion pod area infected by CPRD was estimated as lesion developed on the pod which measured based on the ellipse shape model described in Campbell et al. (2015) and Ling et al. (2017) while the pod surface was measured using the prolate spheroid model described in Ten Hoopen et al. (2012) and Ling et al. (2017).

\section{Fitting disease progress curve}

Four nonlinear models also known as growth-curve models were used to describe the disease progress curve for the proportion pod area infected by CPRD such as exponential model, monomolecular model, logistic model and Gompertz model. The function for four nonlinear models was described in details in Ling et al. (2017).

\section{Goodness of fit test}

Curve fitting on the black pod disease severity was done using PROC NLIN using numerical method of Levenburg-Marquardt to minimize the error sum of squares of fitted models (SAS Institute, 2013).

In order to identify the best fitted model among the four nonlinear models, two goodness of fit tests were used, namely the Akaike Information Criterion (AIC) and the Bayesian Information Criterion (BIC) (Aho et al., 2014). The best model was selected based on the smallest value of AIC and BIC with both functions given in Ling et al. (2017).

\section{Estimating area under disease progress curve}

The area under disease progress curve (AUDPC) was calculated for each nonlinear model fitted to the disease progress curve using the method of Yeh (Simko \& Piepho, 2012; Ling et al., 2017) based on the trapezoidal rule that approximate the area under a curve by dividing the area into a number of strips of equal width.

\section{Grouping genotypes into CPRD tolerant categories}

Two disease severity variables of the AUDPC values and lesion area development rate were used in grouping the cocoa genotypes. Both variables in all cocoa genotypes were grouped based on four tolerance level against the CPRD using $k$-means clustering method with $k=4$. The four different tolerance groups, i.e. tolerant (Group I), moderately tolerant (Group II), moderately susceptible (Group III) and susceptible (Group IV) which similar to Janani \& Kumar (2014) who used resistant instead of tolerant. PROC FASTCLUS (SAS Institute, 2013) was used to run the $k$-means clustering method that involved four steps as described in Jain (2010). PROC CANDISC and PROC SGPLOT procedures were used to obtain a graphical check on the distribution of the clusters (SAS Institute, 2013). 


\section{Statistical analysis}

The disease severity data were subjected to SQRT $(X+0.5)$ transformation before ANOVA test applied. Spearman rank correlation analyses in PROC CORR (SAS Institute, 2013) was used to assess the genotype's tolerance level against CPRD between days after inoculation.

\section{RESULTS AND DISCUSSION}

\section{Genotypes effect on disease severity}

ANOVA test on the proportion of pod infected by CPRD from 1 to 7 days after inoculation (Table 1) showed there was an effect of genotypes on the proportion of pod infected by CPRD inoculation. The mean proportion of pod infected by CPRD observed among the genotypes varied significantly $(\mathrm{P}<0.05)$ from 4 to 7 days of inoculation. This indicated that each genotype available in Malaysia have different tolerant against the CPRD and its effect of tolerance can be seen clearly over longer period of observation from the day of inoculation. Thus, the observation on the proportion pod infected by CPRD in Figure 1 developed slowly for the first 4 days after inoculation and only clearly expanded starting 5 days after inoculation.

The coefficients of rank correlation between 4,5 , and 6 days after inoculations to 7 days after inoculation were significant $(\mathrm{P}<0.05)$ with higher correlation $(r=0.96)$

Table 1. ANOVA test on cocoa genotype effect to proportion pod area infected by CPRD

\begin{tabular}{lcccccccc}
\hline \multirow{2}{*}{$\begin{array}{l}\text { Sources of } \\
\text { variation }\end{array}$} & $\begin{array}{l}\text { Degree of } \\
\text { Freedom }\end{array}$ & 1 DAI & 2 DAI & 3 DAI & 4 DAI & 5 DAI & 6 DAI & 7 DAI \\
\hline Genotype & 49 & $0.0000 \mathrm{~ns}$ & $0.0000 \mathrm{~ns}$ & $0.0001 \mathrm{~ns}$ & $0.0017 *$ & $0.0156^{*}$ & $0.0840^{*}$ & $0.2738^{*}$ \\
Error & 1950 & 0.0000 & 0.0000 & 0.0022 & 0.0229 & 0.1786 & 0.8264 & 2.5143 \\
Total & 1999 & 0.0000 & 0.0000 & 0.0023 & 0.0245 & 0.1942 & 0.9104 & 2.7881 \\
\hline
\end{tabular}

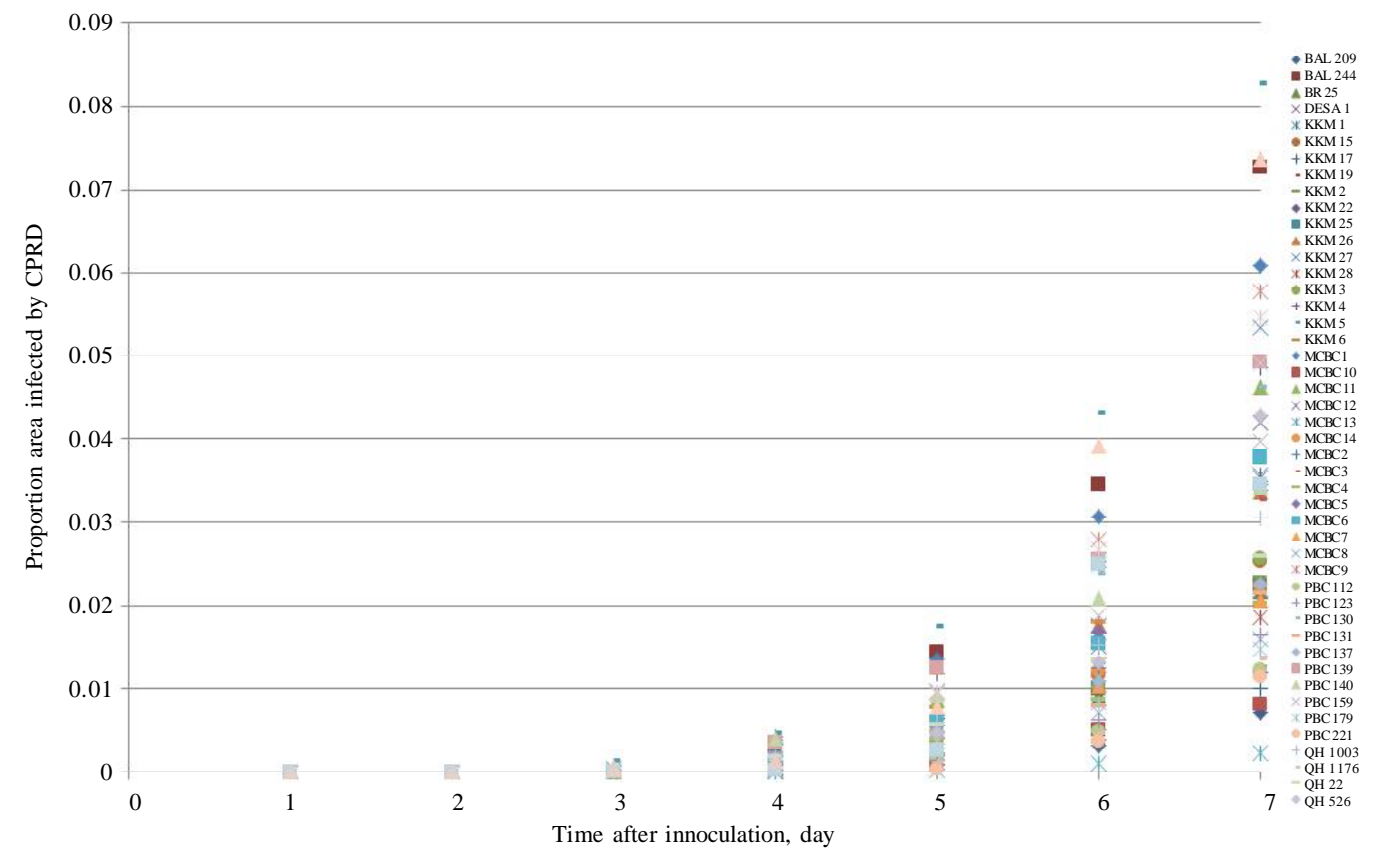

Figure 1. Mean disease severity observed from 1 to 7 days after inoculation for 50 cocoa genotypes 


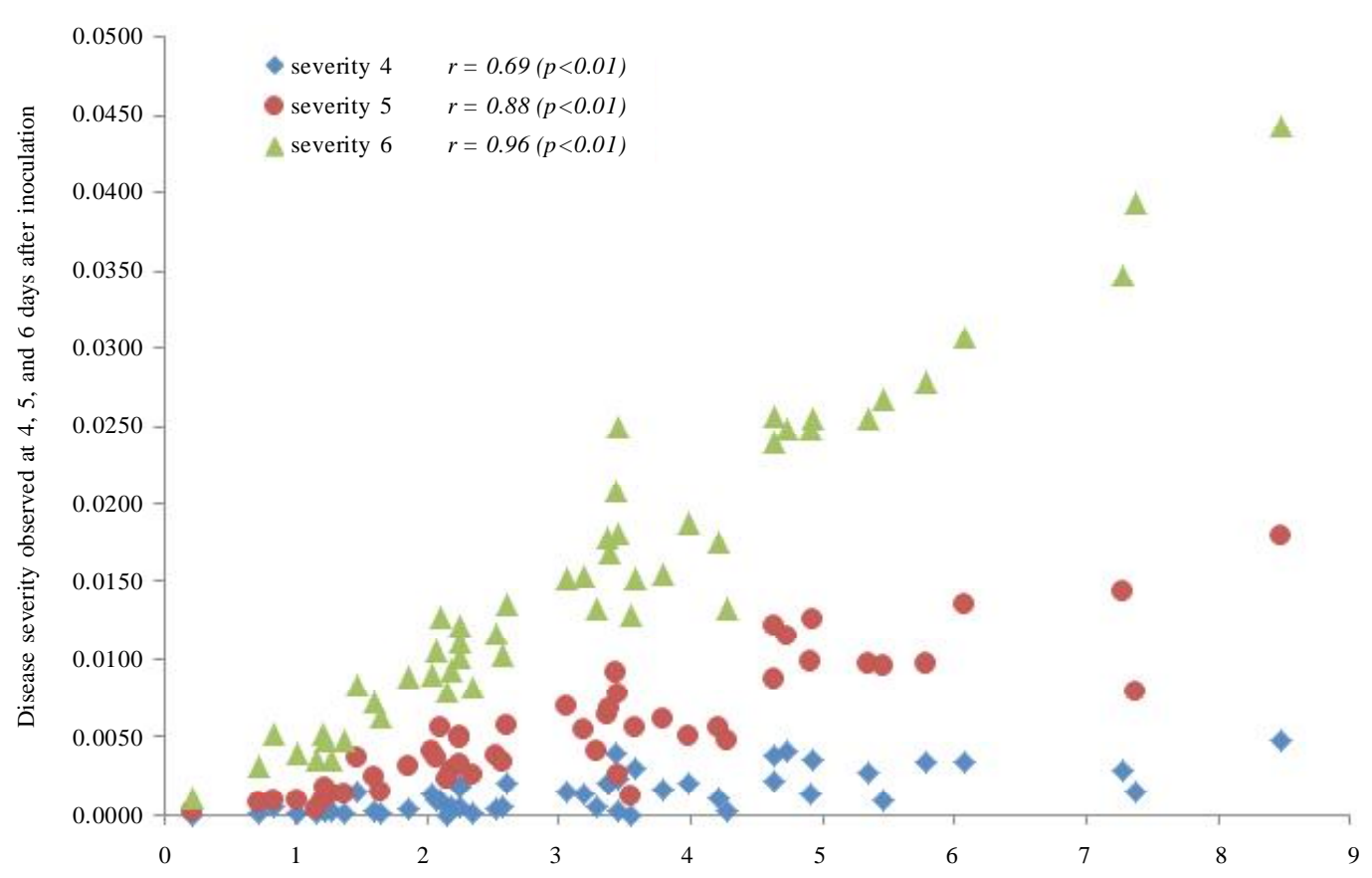

Figure 2. Correlation of disease severity observed between 4 to 7 days after inoculation for 50 cocoa genotypes

was observed between 6 and 7 days after inoculations. This suggests that the growth rate of pod area infected by CPRD getting consistent among the genotypes over time. Efombagn et al. (2011) also recorded that observation on the lesion development using the leaf disc test showed high correlation between 5 and 7 days after inoculation.

However, the disease severity used in grouping the genotype's tolerance level against CPRD was based on the proportion pod infected by CPRD from 1 to 7 days after inoculation because some genotypes have showed lesion symptom on the pod at 1 day after inoculation.

\section{Disease severity curve fitted model}

The overall $F$-value for 50 cocoa genotypes were significant at 5\% level in all developed models except the Monomolecular model in the genotype KKM 19, MCBC12 and PBC 221
(Table 2). Table 3 showed the values of AIC and BIC of four nonlinear models fitted on the disease severity of each genotype. The results of AIC test and BIC test showed the Gompertz model fitted well the data disease severity in 47 genotypes with the smallest value in AIC and BIC compared to Monomolecular, Exponential and Logistic models. Another three genotypes, QH 326, MCBC 6 and KKM 1 were fitted well with the Logistic model. This can be seen in the example of predictive curves for four nonlinear models on two control genotypes that tolerant (PBC 123) and susceptible (KKM 4) against CPRD showed three nonlinear (Exponential, Logistic and Grompertz) models fitted well to severity data except Monomolecular model (Figure 3). As only one model need to be selected to represent the CPRD severity progress curve, the Gompertz model was chosen due to higher percentage of Gompertz model fitted well to the data compare to the Logistic model. The application of Gompertz model 
Table 2. Nonlinear models fitted on CPRD progress curves

\begin{tabular}{|c|c|c|c|c|c|c|c|c|c|}
\hline Genotype & Model & $F$ value & AIC & $\mathrm{BIC}$ & Genotype & Model & F value & AIC & $\mathrm{BIC}$ \\
\hline BAL 209 & $\begin{array}{l}\text { Exp. } \\
\text { Gomp. } \\
\text { Log. } \\
\text { Mono. }\end{array}$ & $\begin{array}{r}533.17 * * \\
1418.74 * * \\
539.2 * * \\
8.23 *\end{array}$ & $\begin{array}{r}-95.49 \\
-102.32 \\
-95.57 \\
-68.12\end{array}$ & $\begin{array}{r}-95.60 \\
-102.43 \\
-95.68 \\
-68.23\end{array}$ & MCBC 3 & $\begin{array}{l}\text { Exp. } \\
\text { Gomp. } \\
\text { Log. } \\
\text { Mono. }\end{array}$ & $\begin{array}{rl}797.92 & * * \\
4296.27 & * * \\
848.82 & * * \\
7.28 & *\end{array}$ & $\begin{array}{l}-76.80 \\
-88.56 \\
-77.23 \\
-45.96\end{array}$ & $\begin{array}{l}-76.91 \\
-88.67 \\
-77.34 \\
-46.07\end{array}$ \\
\hline BAL 244 & $\begin{array}{l}\text { Exp. } \\
\text { Gomp. } \\
\text { Log. } \\
\text { Mono. }\end{array}$ & $\begin{array}{r}622.55 * * \\
8568.55 * * \\
728.28 * * \\
10.65 *\end{array}$ & $\begin{array}{l}-63.39 \\
-81.71 \\
-64.48 \\
-36.36\end{array}$ & $\begin{array}{l}-63.49 \\
-81.82 \\
-64.59 \\
-36.46\end{array}$ & MCBC 4 & $\begin{array}{l}\text { Exp. } \\
\text { Gomp. } \\
\text { Log. } \\
\text { Mono. }\end{array}$ & $\begin{array}{c}1079.99 * * \\
3952.97 * * \\
1127.56 * * \\
10.82 *\end{array}$ & $\begin{array}{l}-85.49 \\
-94.56 \\
-85.79 \\
-54.70\end{array}$ & $\begin{array}{l}-85.59 \\
-94.66 \\
-85.89 \\
-54.81\end{array}$ \\
\hline BR 25 & $\begin{array}{l}\text { Exp. } \\
\text { Gomp. } \\
\text { Log. } \\
\text { Mono. }\end{array}$ & $\begin{array}{r}2139.36 * * \\
20054.60 * * \\
2304.96 * * \\
8.51 *\end{array}$ & $\begin{array}{r}-89.26 \\
-104.91 \\
-89.78 \\
-52.36\end{array}$ & $\begin{array}{r}-89.36 \\
-105.02 \\
-89.89 \\
-52.47\end{array}$ & MCBC 5 & $\begin{array}{l}\text { Exp. } \\
\text { Gomp. } \\
\text { Log. } \\
\text { Mono. }\end{array}$ & $\begin{array}{rl}599.95 & * * \\
4714.30 & * * \\
644.91 & * * \\
12.07 & *\end{array}$ & $\begin{array}{l}-73.67 \\
-88.08 \\
-74.18 \\
-47.62\end{array}$ & $\begin{array}{l}-73.78 \\
-88.19 \\
-74.29 \\
-47.73\end{array}$ \\
\hline DESA 1 & $\begin{array}{l}\text { Exp. } \\
\text { Gomp. } \\
\text { Log. } \\
\text { Mono. }\end{array}$ & $\begin{array}{c}1446.55 * * \\
12750.4 * * \\
1618.91 * * \\
8.16 *\end{array}$ & $\begin{array}{l}-77.49 \\
-92.71 \\
-78.27 \\
-43.10\end{array}$ & $\begin{array}{l}-77.60 \\
-92.82 \\
-78.38 \\
-43.21\end{array}$ & MCBC 6 & $\begin{array}{l}\text { Exp. } \\
\text { Gomp. } \\
\text { Log. } \\
\text { Mono. }\end{array}$ & $\begin{array}{c}4071.47 * * \\
2361.83 * * \\
4491.77 * * \\
8.79 *\end{array}$ & $\begin{array}{l}-86.13 \\
-82.32 \\
-86.82 \\
-44.91\end{array}$ & $\begin{array}{l}-86.24 \\
-82.43 \\
-86.93 \\
-45.02\end{array}$ \\
\hline KKM 1 & $\begin{array}{l}\text { Exp. } \\
\text { Gomp. } \\
\text { Log. } \\
\text { Mono. }\end{array}$ & $\begin{array}{c}2509.74 * * \\
1385.61 * * \\
2623.40 * * \\
9.92 *\end{array}$ & $\begin{array}{l}-83.36 \\
-79.21 \\
-83.67 \\
-46.19\end{array}$ & $\begin{array}{l}-83.47 \\
-79.31 \\
-83.78 \\
-46.30\end{array}$ & MCBC 7 & $\begin{array}{l}\text { Exp. } \\
\text { Gomp. } \\
\text { Log. } \\
\text { Mono. }\end{array}$ & $\begin{array}{r}358.80 * * \\
1184.81 * * \\
371.64 * * \\
11.77 *\end{array}$ & $\begin{array}{l}-76.75 \\
-85.08 \\
-76.99 \\
-54.13\end{array}$ & $\begin{array}{l}-76.86 \\
-85.19 \\
-77.10 \\
-54.24\end{array}$ \\
\hline KKM 15 & $\begin{array}{l}\text { Exp. } \\
\text { Gomp. } \\
\text { Log. } \\
\text { Mono. }\end{array}$ & $\begin{array}{c}303.89 * * \\
838.88 * * \\
314.51 * * \\
9.22 *\end{array}$ & $\begin{array}{l}-73.46 \\
-80.54 \\
-73.70 \\
-50.62\end{array}$ & $\begin{array}{l}-73.57 \\
-80.64 \\
-73.81 \\
-50.73\end{array}$ & MCBC 8 & $\begin{array}{l}\text { Exp. } \\
\text { Gomp. } \\
\text { Log. } \\
\text { Mono. }\end{array}$ & $\begin{array}{r}430.42 * * \\
1300.05 * * \\
441.13 * * \\
7.97 *\end{array}$ & $\begin{array}{l}-82.41 \\
-90.12 \\
-82.58 \\
-56.35\end{array}$ & $\begin{array}{l}-82.52 \\
-90.23 \\
-82.69 \\
-56.46\end{array}$ \\
\hline KKM 17 & $\begin{array}{l}\text { Exp. } \\
\text { Gomp. } \\
\text { Log. } \\
\text { Mono. }\end{array}$ & $\begin{array}{r}595.07 * * \\
1917.90 * * \\
605.14 * * \\
7.05 *\end{array}$ & $\begin{array}{l}-91.47 \\
-99.64 \\
-91.58 \\
-62.51\end{array}$ & $\begin{array}{l}-91.57 \\
-99.75 \\
-91.69 \\
-62.62\end{array}$ & MCBC 9 & $\begin{array}{l}\text { Exp. } \\
\text { Gomp. } \\
\text { Log. } \\
\text { Mono. }\end{array}$ & $\begin{array}{rl}643.07 & * * \\
8743.41 & * * \\
732.12 & * * \\
10.51 & *\end{array}$ & $\begin{array}{l}-66.85 \\
-85.09 \\
-67.75 \\
-39.52\end{array}$ & $\begin{array}{l}-66.96 \\
-85.20 \\
-67.86 \\
-39.62\end{array}$ \\
\hline KKM 19 & $\begin{array}{l}\text { Exp. } \\
\text { Gomp. } \\
\text { Log. } \\
\text { Mono. }\end{array}$ & $\begin{array}{r}2807.70 * * \\
16616.30 * * \\
2905.69 * * \\
5.57 \mathrm{~ns}\end{array}$ & $\begin{array}{r}-99.06 \\
-111.50 \\
-99.30 \\
-58.09\end{array}$ & $\begin{array}{r}-99.17 \\
-111.61 \\
-99.41 \\
-58.20\end{array}$ & PBC 112 & $\begin{array}{l}\text { Exp. } \\
\text { Gomp. } \\
\text { Log. } \\
\text { Mono. }\end{array}$ & $\begin{array}{r}596.98 * * \\
1340.05 * * \\
607.01 * * \\
8.50 *\end{array}$ & $\begin{array}{l}-88.75 \\
-94.39 \\
-88.87 \\
-60.77\end{array}$ & $\begin{array}{l}-88.86 \\
-94.50 \\
-88.97 \\
-60.87\end{array}$ \\
\hline KKM 2 & $\begin{array}{l}\text { Exp. } \\
\text { Gomp. } \\
\text { Log. } \\
\text { Mono. }\end{array}$ & $\begin{array}{c}1405.10 * * \\
1873.72 * * \\
1444.80 * * \\
6.83 *\end{array}$ & $\begin{array}{l}-86.00 \\
-88.01 \\
-86.19 \\
-50.88\end{array}$ & $\begin{array}{l}-86.10 \\
-88.12 \\
-86.30 \\
-50.99\end{array}$ & PBC 123 & $\begin{array}{l}\text { Exp. } \\
\text { Gomp. } \\
\text { Log. } \\
\text { Mono. }\end{array}$ & $\begin{array}{rl}512.01 & * * \\
1501.21 & * * \\
523.90 & * * \\
6.13 & *\end{array}$ & $\begin{array}{l}-83.99 \\
-91.50 \\
-84.15 \\
-55.37\end{array}$ & $\begin{array}{l}-84.10 \\
-91.61 \\
-84.26 \\
-55.48\end{array}$ \\
\hline KKM 22 & $\begin{array}{l}\text { Exp. } \\
\text { Gomp. } \\
\text { Log. } \\
\text { Mono. }\end{array}$ & $\begin{array}{c}379.71 * * \\
925.20 * * \\
385.95 * * \\
6.95 *\end{array}$ & $\begin{array}{l}-85.72 \\
-91.93 \\
-85.84 \\
-59.82\end{array}$ & $\begin{array}{l}-85.83 \\
-92.04 \\
-85.94 \\
-59.93\end{array}$ & PBC 130 & $\begin{array}{l}\text { Exp. } \\
\text { Gomp. } \\
\text { Log. } \\
\text { Mono. }\end{array}$ & $\begin{array}{r}746.97 * * \\
6001.21 * * \\
830.52 * * \\
15.58 * *\end{array}$ & $\begin{array}{l}-70.69 \\
-85.26 \\
-71.43 \\
-44.62\end{array}$ & $\begin{array}{l}-70.80 \\
-85.37 \\
-71.54 \\
-44.73\end{array}$ \\
\hline KKM 25 & $\begin{array}{l}\text { Exp. } \\
\text { Gomp. } \\
\text { Log. } \\
\text { Mono. }\end{array}$ & $\begin{array}{r}699.04 * * \\
3339.51 * * \\
731.77 * * \\
9.20 *\end{array}$ & $\begin{array}{l}-81.22 \\
-92.14 \\
-81.53 \\
-52.56\end{array}$ & $\begin{array}{l}-81.32 \\
-92.25 \\
-81.64 \\
-52.67\end{array}$ & PBC131 & $\begin{array}{l}\text { Exp. } \\
\text { Gomp. } \\
\text { Log. } \\
\text { Mono. }\end{array}$ & $\begin{array}{c}1003.60 * * \\
6951.23 * * \\
1049.16 * * \\
6.42 *\end{array}$ & $\begin{array}{l}-84.25 \\
-97.79 \\
-84.56 \\
-51.18\end{array}$ & $\begin{array}{l}-84.36 \\
-97.89 \\
-84.67 \\
-51.28\end{array}$ \\
\hline KKM 26 & $\begin{array}{l}\text { Exp. } \\
\text { Gomp. } \\
\text { Log. } \\
\text { Mono. }\end{array}$ & $\begin{array}{r}339.92 * * \\
1136.45 * * \\
359.21 * * \\
11.63 *\end{array}$ & $\begin{array}{l}-69.62 \\
-78.03 \\
-70.00 \\
-47.30\end{array}$ & $\begin{array}{l}-69.73 \\
-78.14 \\
-70.11 \\
-47.41\end{array}$ & PBC 137 & $\begin{array}{l}\text { Exp. } \\
\text { Gomp. } \\
\text { Log. } \\
\text { Mono. }\end{array}$ & $\begin{array}{r}813.15 * * \\
3813.18 * * \\
853.42 * * \\
13.76 * *\end{array}$ & $\begin{array}{l}-81.81 \\
-92.61 \\
-82.15 \\
-54.41\end{array}$ & $\begin{array}{l}-81.92 \\
-92.72 \\
-82.26 \\
-54.51\end{array}$ \\
\hline KKM 27 & $\begin{array}{l}\text { Exp. } \\
\text { Gomp. } \\
\text { Log. } \\
\text { Mono. }\end{array}$ & $\begin{array}{r}652.39 * * \\
9323.24 * * \\
735.49 * * \\
11.23 *\end{array}$ & $\begin{array}{l}-68.13 \\
-86.73 \\
-68.97 \\
-41.08\end{array}$ & $\begin{array}{l}-68.24 \\
-86.83 \\
-69.08 \\
-41.19\end{array}$ & PBC 139 & $\begin{array}{l}\text { Exp. } \\
\text { Gomp. } \\
\text { Log. } \\
\text { Mono. }\end{array}$ & $\begin{array}{r}357.56 * * \\
1330.79 * * \\
385.90 * * \\
14.52 * *\end{array}$ & $\begin{array}{l}-64.64 \\
-73.80 \\
-65.17 \\
-43.28\end{array}$ & $\begin{array}{l}-64.75 \\
-73.91 \\
-65.28 \\
-43.38\end{array}$ \\
\hline KKM 28 & $\begin{array}{l}\text { Exp. } \\
\text { Gomp. } \\
\text { Log. } \\
\text { Mono. }\end{array}$ & $\begin{array}{c}323.75 * * \\
859.95 * * \\
332.46 * * \\
9.11 *\end{array}$ & $\begin{array}{l}-77.78 \\
-84.59 \\
-77.97 \\
-54.43\end{array}$ & $\begin{array}{l}-77.89 \\
-84.70 \\
-78.08 \\
-54.54\end{array}$ & PBC 140 & $\begin{array}{l}\text { Exp. } \\
\text { Gomp. } \\
\text { Log. } \\
\text { Mono. }\end{array}$ & $\begin{array}{r}288.07 * * \\
895.65 * * \\
304.61 * * \\
19.41 * *\end{array}$ & $\begin{array}{l}-67.71 \\
-75.61 \\
-68.09 \\
-49.61\end{array}$ & $\begin{array}{l}-67.81 \\
-75.71 \\
-68.20 \\
-49.72\end{array}$ \\
\hline
\end{tabular}




\begin{tabular}{|c|c|c|c|c|c|c|c|c|c|}
\hline \multirow[t]{4}{*}{ KKM 3} & Exp. & $2527.07 * *$ & -88.23 & -88.34 & \multirow[t]{4}{*}{ PBC 159} & Exp. & $432.41 * *$ & -69.41 & -69.51 \\
\hline & Gomp. & $16131.00 * *$ & -101.20 & -101.31 & & Gomp. & $1367.50 * *$ & -77.44 & -77.55 \\
\hline & Log. & $2767.09 * *$ & -88.87 & -88.98 & & Log. & $461.14 * *$ & -69.85 & -69.96 \\
\hline & Mono. & $7.60 *$ & -49.57 & -49.68 & & Mono. & $9.21 *$ & -44.10 & -44.21 \\
\hline \multirow[t]{4}{*}{ KKM 4} & Exp. & $895.05 * *$ & -71.18 & -71.29 & \multirow[t]{4}{*}{ PBC 179} & Exp. & $368.36 * *$ & -81.40 & -81.50 \\
\hline & Gomp. & $10115.80 * *$ & -88.14 & -88.25 & & Gomp. & $1017.10 * *$ & -88.48 & -88.58 \\
\hline & Log. & $1014.51 * *$ & -72.06 & -72.16 & & Log. & $377.08 * *$ & -81.56 & -81.67 \\
\hline & Mono. & $14.47 * *$ & -43.40 & -43.51 & & Mono. & $15.12 * *$ & -60.07 & -60.18 \\
\hline \multirow[t]{4}{*}{ KKM 5} & Exp. & $538.52 * *$ & -60.33 & -60.44 & \multirow[t]{4}{*}{ PBC 221} & Exp. & $291.44 * *$ & -84.31 & -84.42 \\
\hline & Gomp. & $10652.80 * *$ & -81.19 & -81.30 & & Gomp. & $517.02 * *$ & -88.29 & -88.40 \\
\hline & Log. & $647.82 * *$ & -61.62 & -61.73 & & Log. & $294.44 * *$ & -84.38 & -84.49 \\
\hline & Mono. & $12.24 *$ & -35.11 & -35.21 & & Mono. & $4.85 \mathrm{~ns}$ & -58.49 & -58.59 \\
\hline \multirow[t]{4}{*}{ KKM 6} & Exp. & $398.08 * *$ & -70.59 & -70.70 & \multirow[t]{4}{*}{ QH 1003} & Exp. & $450.65 * *$ & -73.03 & -73.13 \\
\hline & Gomp. & $1774.35 * *$ & -81.02 & -81.12 & & Gomp. & $1352.46 * *$ & -80.69 & -80.80 \\
\hline & Log. & $422.62 * *$ & -71.00 & -71.11 & & Log. & $472.34 * *$ & -73.35 & -73.46 \\
\hline & Mono. & $13.53 * *$ & -48.06 & -48.17 & & Mono. & $11.63 *$ & -48.75 & -48.86 \\
\hline \multirow[t]{4}{*}{ MCBC 1} & Exp. & $470.43 * *$ & -63.74 & -63.85 & \multirow[t]{4}{*}{ QH 1176} & Exp. & $811.59 * *$ & -89.15 & -89.25 \\
\hline & Gomp. & $3760.74 * *$ & -78.26 & -78.37 & & Gomp. & $3460.02 * *$ & -99.28 & -99.39 \\
\hline & Log. & $529.02 * *$ & -64.56 & -64.67 & & Log. & $831.96 * *$ & -89.32 & -89.43 \\
\hline & Mono. & $11.99 *$ & -39.35 & -39.45 & & Mono. & $6.07 *$ & -57.27 & -57.38 \\
\hline \multirow[t]{4}{*}{ MCBC 10} & Exp. & $75.95 * *$ & -79.09 & -79.20 & \multirow[t]{4}{*}{ QH 22} & Exp. & $709.75 * *$ & -78.53 & -78.64 \\
\hline & Gomp. & $105.92 * *$ & -81.35 & -81.46 & & Gomp. & $3835.78 * *$ & -90.32 & -90.43 \\
\hline & Log. & $76.40 * *$ & -79.13 & -79.24 & & Log. & $749.92 * *$ & -78.91 & -79.02 \\
\hline & Mono. & $9.79 *$ & -66.11 & -66.22 & & Mono. & $13.43 * *$ & -51.93 & -52.04 \\
\hline \multirow[t]{4}{*}{ MCBC 11} & Exp. & $215.45 * *$ & -61.99 & -62.10 & \multirow[t]{4}{*}{ QH 326} & Exp. & $1927.52 * *$ & -79.65 & -79.76 \\
\hline & Gomp. & $611.87 * *$ & -69.24 & -69.35 & & Gomp. & $1045.50 * *$ & -75.38 & -75.49 \\
\hline & Log. & $229.30 * *$ & -62.42 & -62.53 & & Log. & $1929.25 * *$ & -79.66 & -79.77 \\
\hline & Mono. & $12.22 *$ & -43.13 & -43.23 & & Mono. & $5.96 *$ & -41.64 & -41.75 \\
\hline \multirow[t]{4}{*}{ MCBC 12} & Exp. & $307.86 * *$ & -69.53 & -69.64 & \multirow[t]{4}{*}{ QH 37} & Exp. & $37.58 * *$ & -53.46 & -53.56 \\
\hline & Gomp. & $729.19 * *$ & -75.53 & -75.64 & & Gomp. & $50.44 * *$ & -55.40 & -55.51 \\
\hline & Log. & $319.25 * *$ & -69.78 & -69.89 & & Log. & $38.27 * *$ & -53.58 & -53.68 \\
\hline & Mono. & $5.54 \mathrm{~ns}$ & -43.96 & -44.07 & & Mono. & $9.04 *$ & -44.74 & -44.85 \\
\hline \multirow[t]{4}{*}{ MCBC 13} & Exp. & $79.78 * *$ & -99.66 & -99.76 & \multirow[t]{4}{*}{ QH 441} & Exp. & $156.20 * *$ & -53.48 & -53.59 \\
\hline & Gomp. & $100.62 * *$ & -101.24 & -101.34 & & Gomp. & $369.80 * *$ & -59.45 & -59.56 \\
\hline & Log. & $79.87 * *$ & -99.66 & -99.77 & & Log. & $168.71 * *$ & -54.01 & -54.12 \\
\hline & Mono. & $7.99 *$ & -83.96 & -84.07 & & Mono. & $8.61 *$ & -34.87 & -34.97 \\
\hline \multirow[t]{4}{*}{ MCBC 14} & Exp. & $431.44 * *$ & -77.15 & -77.26 & \multirow[t]{4}{*}{ QH 968} & Exp. & $311.31 * *$ & -63.96 & -64.07 \\
\hline & Gomp. & $1664.98 * *$ & -86.57 & -86.68 & & Gomp. & $1148.58 * *$ & -73.06 & -73.16 \\
\hline & Log. & $449.47 * *$ & -77.43 & -77.54 & & Log. & $334.94 * *$ & -64.47 & -64.57 \\
\hline & Mono. & $14.69 * *$ & -54.55 & -54.66 & & Mono. & $11.07 *$ & -41.97 & -42.08 \\
\hline \multirow[t]{4}{*}{ MCBC 2} & Exp. & $148.42 * *$ & -69.86 & -69.97 & \multirow[t]{4}{*}{ RP 1} & Exp. & $413.88 * *$ & -64.47 & -64.58 \\
\hline & Gomp. & $294.93 * *$ & -74.61 & -74.72 & & Gomp. & $1815.58 * *$ & -74.79 & -74.90 \\
\hline & Log. & $152.01 * *$ & -70.03 & -70.13 & & Log. & $453.73 * *$ & -65.11 & -65.22 \\
\hline & Mono. & $16.42 * *$ & -55.33 & -55.43 & & Mono. & $10.30 *$ & -40.10 & -40.20 \\
\hline
\end{tabular}

in describing the disease progress also supported by Berger (1981) who reported that Gompertz model was better fit to other statistical models in plant diseases including estimation of epidemic rate, projection of future disease severity and determination of initial disease. Besides that, the Gompertz model was able to linearize the asymmetrical disease progress curves which happened to many pathosystems known as polycyclic disease compared to the Logistic model (Berger, 1981; Sastry \& Zitter, 2014). The exponential model was not selected in fitting the disease severity because the model was more appropriate to describe the very early stages of most polycyclic epidemics but not efficient in growth stage (Contreras-Medina et al., 2009). 

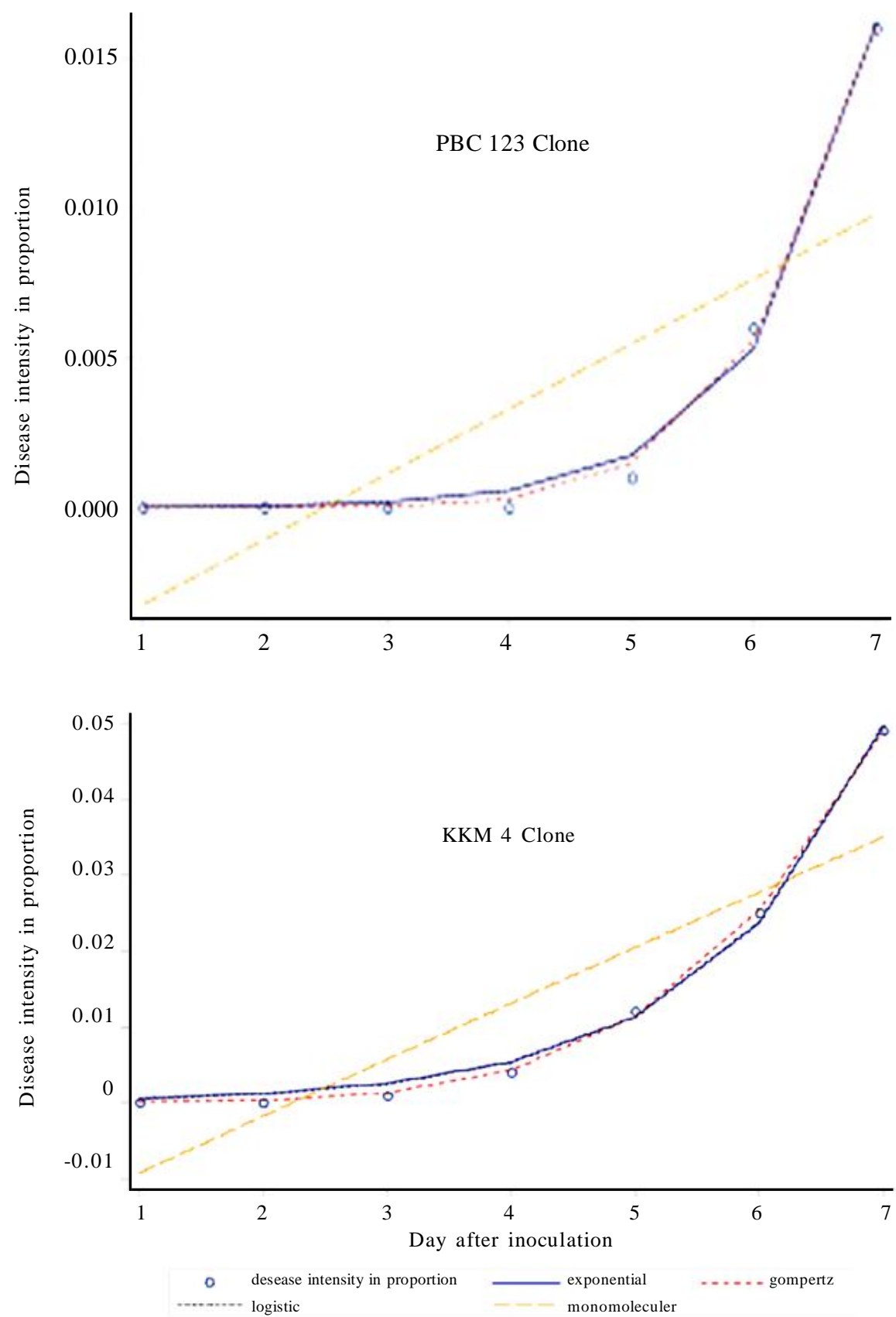

Figure 3. CPRD progress curve fitted with four different nonlinear models on control cocoa genotypes PBC 123 (top) and KKM 4 (bottom) 


\section{Estimate area under disease progress curve}

The AUDPC has been widely used in two major areas such as crop loss assessment and field assessment of quantitative resistance as it integrate all aspects of disease progress related to host development and growth (Mau \& Ndiwa, 2018). It is helpful to plot the proportion pod infected versus the day after inoculation ("disease progress curve") to get a better idea of how genotypes perform in the experiment. For example, Figure 4 showed the cummulative AUDPC of CPRD in two control genotypes (KKM 4 and PBC 123) of different tolerant level inoculated with the P. palmivora. The plot showed KKM 4 as susceptible genotype has larger AUDPC compared to PBC 123 as tolerant genotype. This could be explained by the faster infection rate in the susceptible genotype with increasing in the slope of the curve compared to tolerant genotype over time. The higher the AUDPC, the more disease or susceptible in the genotype (Forbes et al., 2014). Meanwhile, Figure 5 showed the estimated AUDPC from CPRD severity disease progress curve fitted with Gompertz models for 50 genotypes. Result in Figure 5 showed the AUDPC values ranged from 2.246 to 13.194 with eight genotypes having the lowest AUDPC values compared to PBC123, i.e. MCBC 13 (2.246), PBC 221 (3.827), BAL 209 (3.992), KKM 19 (4.075), KKM 17 (4.297), QH 1176 (4.52) and KKM 22 (4.65) while KKM 5 (13.194), BAL 244 (11.809), MCBC 1 (11.550), PBC139 (11.317) and QH 441 (11.290) have the highest AUDPC values compared to KKM 4 (11.29).

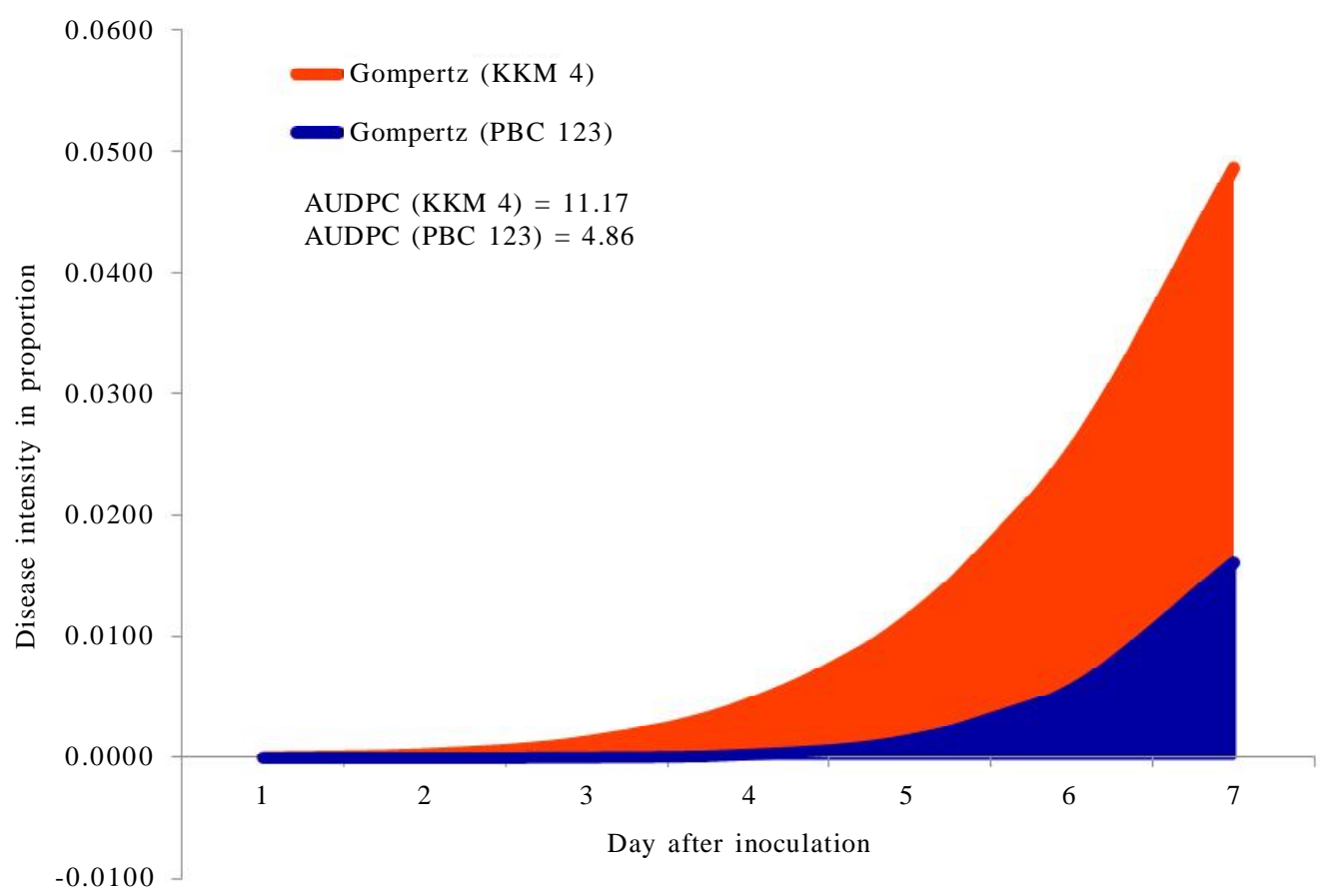

Figure 4. The cumulative AUDPC of CPRD on two cocoa genotypes (KKM 4 and PBC 123) of different tolerance level inoculated with the P. palmivora 


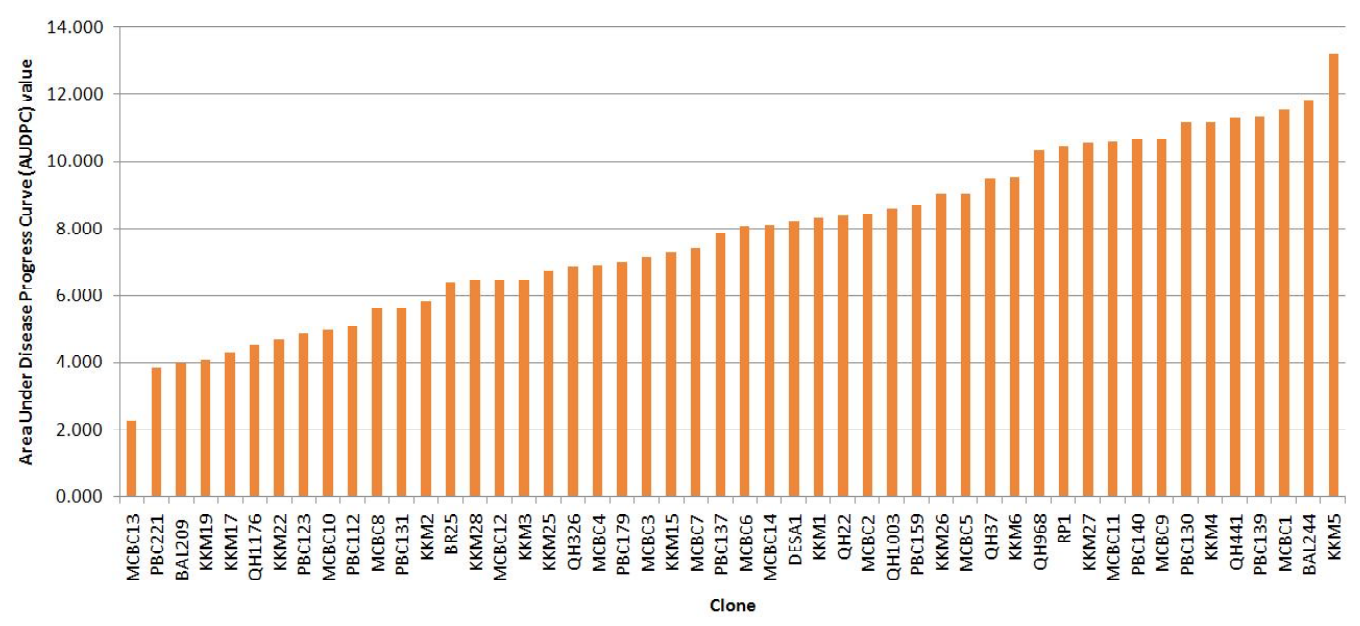

Figure 5. The AUDPC values arranged in ascending order for 50 cocoa genotypes against CPRD

\section{Genotype tolerance groups against CPRD}

The result from the grouping of the $k$-means clustering method in Table 3 showed group I (tolerant) contained 10 genotypes with the rate of lesion area development ranged between 0.079 to $0.804 \mathrm{~cm}^{2} /$ day and the AUDPC was ranged between 2.246 to 5.049 . These genotypes can be considered as potential tolerant as they fall in the same group with tolerant genotypes (PBC123). In addition, there were few genotypes with lower values in the lesion area development rate and AUDPC than PBC 123. The genotypes were MCBC 13, PBC 221, BAL 209, KKM 19, QH 1176 and KKM 22. Some of the genotypes in group I such as KKM 22 and PBC 123 were identified as class I clones which generally suitable for planting throughout Malaysia with potential high yield more than 2.5 ton/ha/year (Lee et al., 2013).

Meanwhile the group II (moderately tolerant) has 14 genotypes with the rate of lesion area development ranged between 0.708 to $1.744 \mathrm{~cm}^{2} /$ day and the AUDPC was ranged between 5.594 to 7.395 . The study also confirmed that cocoa genotype BR 25 previously reported as moderate resistant in Ling et al. (2017) fall in this group. The group III (moderately susceptible) has 13 genotypes with the rate of lesion area development ranged between 0.930 to $1.960 \mathrm{~cm}^{2} /$ day and the AUDPC was ranged between 7.830 to 9.478 . In this group III, there was an inconsistent result of genotype QH 1003 where previous study in Ling et al. (2017) categorised as resistant. This could be due to changes of pathogenicity of the P. palmivora as also reported by Susilo \& Anita-Sari (2014). For group IV (susceptible), there was 13 genotypes with the rate of lesion area development ranged between 1.846 to $3.149 \mathrm{~cm}^{2} /$ day and the AUDPC was ranged between 10.339 to 13.194. These genotypes was considered to be susceptible as they fall in the same group with susceptible genotype (KKM 4) used as control in this study. The plot of cluster distribution in Figure 6 suggests that 4 groups seem to be able to partition the genotypes clearly with the centroids for four groups are slightly different. 
Table 3. Clustering the 50 cocoa genotypes based on the rate of lesion area development and AUDPC

\begin{tabular}{|c|c|c|c|}
\hline \multirow[b]{2}{*}{ Groups } & \multicolumn{2}{|c|}{ Disease severity measure } & \multirow[b]{2}{*}{ Genotypes } \\
\hline & $\begin{array}{c}\text { Rate of lesion area } \\
\text { development, } \mathrm{cm}^{2} / \text { day* }\end{array}$ & AUDPC* & \\
\hline I & $0.512(0.079-0.804)$ & $4.247(2.246-5.049)$ & $\begin{array}{l}\text { MCBC 13; PBC 221; BAL 209; KKM 19; } \\
\text { KKM 17; QH 1176; KKM 22; PBC 123; } \\
\text { MCBC 10; PBC } 112\end{array}$ \\
\hline II & $1.079(0.708-1.744)$ & $6.562(5.594-7.395)$ & $\begin{array}{l}\text { MCBC 8; PBC 131; KKM 2; BR 25; } \\
\text { KKM 28; MCBC 12; KKM 3; KKM 25; } \\
\text { QH 326; MCBC 4; PBC 179; MCB C3; } \\
\text { KKM } 15 ; \text { MCBC } 7\end{array}$ \\
\hline III & $1.511(0.930-1.960)$ & $8.583(7.830-9.478)$ & $\begin{array}{l}\text { PBC 137; MCBC 6; MCBC 14; DESA 1; } \\
\text { KKM 1; QH 22; MCBC 2; QH 1003; } \\
\text { PBC 159; KKM 26; MCBC 5; QH 37; } \\
\text { KKM } 6\end{array}$ \\
\hline IV & $2.517(1.846-3.149)$ & $11.133(10.339-13.194)$ & $\begin{array}{l}\text { QH 968; RP 1; KKM 27; MCBC } 11 ; \text { PBC 140; } \\
\text { MCBC 9; PBC 130; KKM 4; QH 441; } \\
\text { PBC 139; MCBC 1; BAL 244; KKM } 5\end{array}$ \\
\hline
\end{tabular}

Note: * The number in the bracket is the range value

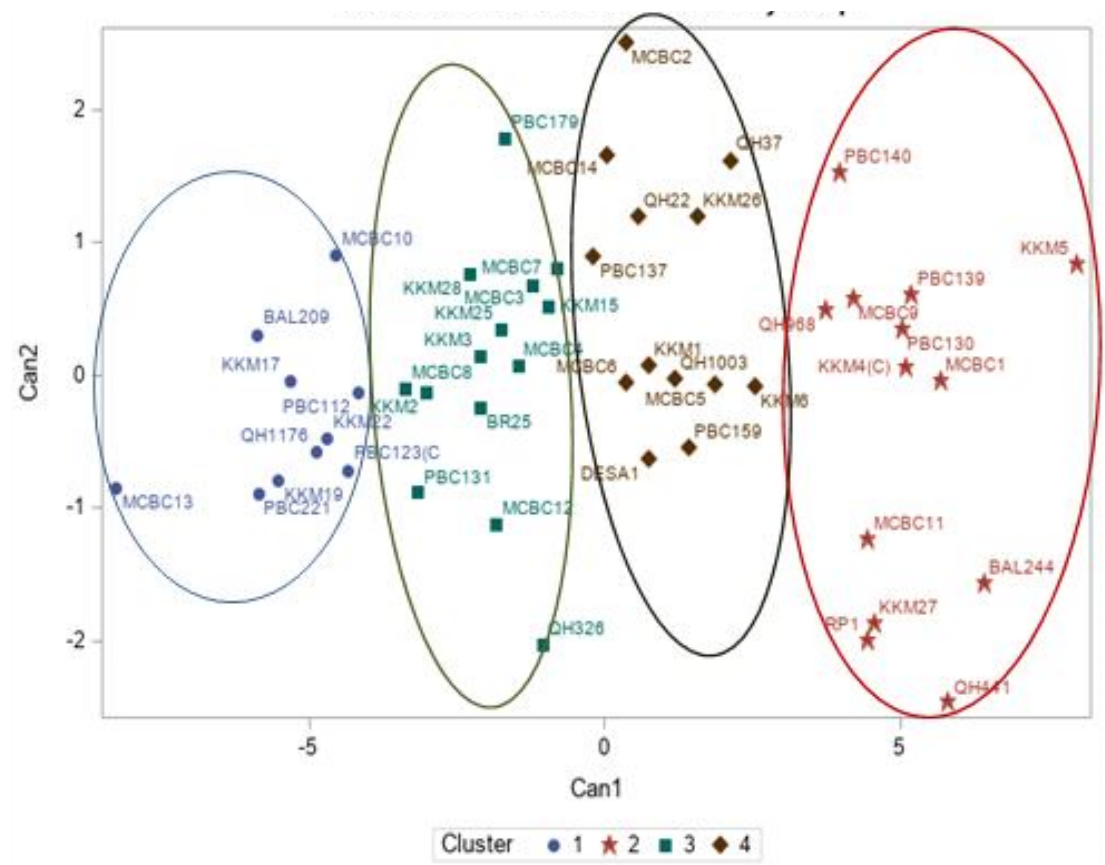

Figure 6. An example of the resulting plot canonical variables identified by group PROC FASTCLUS 


\section{CONCLUSIONS}

In conclusion, the developed screening method has identified the proportion pod infected by CPRD was well-fitted with Gompertz models over the 50 genotypes planted in Malaysia and used in estimating the AUDPC. The significant different in response to the tolerance level against CPRD based on the proportion pod infected starting 4 days after inoculation proved there were potential characteristics of tolerance against CPRD among the genotypes. The diversity of tolerance level against CPRD for 50 genotypes were able to be grouped into four groups. There are 10 genotypes were grouped in group I (tolerant), namely MCBC 13, PBC 221, BAL 209, KKM 19 , KKM 17, QH 1176, KKM 22, РBC 123, MCBC 10, and PBC 112. Group II (moderately tolerant) recorded 14 genotypes, namely MCBC 8, PBC 131, KKM 2, BR 25, KKM 28, MCBC 12, KKM 3, KKM 25, QH 326, MCBC 4, PBC 179, MCBC 3, KKM 15 and MCBC 7. Meanwhile group III (moderately susceptible) recorded 13 genotypes, namely PBC 137, MCBC 6, MCBC 14, DESA 1, KKM 1, QH 22, MCBC 2, QH 1003, PBC 159, KKM 26, MCBC 5, QH 37 and KKM 6. Group IV (susceptible) recorded 13 genotypes, namely QH 968, RP1, KKM 27, MCBC 11, PBC 140, MCBC 9, PBC 130, KKM 4, QH441, PBC 139, MCBC 1, BAL 244 and KKM 5. This grouping of 50 genotypes into four tolerant levels enable to guide the breeders and farmers to identify the genotypes to be planted in the field.

\section{ACKNOWLEDGEMENTS}

The authors wish to thank the Director-General of Malaysian Cocoa Board (MCB) and the Director of Upstream Technology Cocoa for permission of publishing and reviewing this paper. Thanks are also due to staffs at Plant Breeding Unit and Plant
Pathology Unit in CRDC Jengka, Pahang, CRDC Bagan Datuk, Perak and CRDC Tawau, Sabah for providing the data used in this study.

\section{REFERENCES}

Aho, K.; D. Derryberry \& T. Peterson (2014). Model selection for ecologists: the worldviews of AIC and BIC. Ecology, 95, 631-636.

Alsultan, W.; G. Vadamalai; A. Khairulmazmi; H. Mohd Saud; A.M. Al-Sadi; O. Rashed; A.K. Mohd Jaaffar \& A. Nasehi (2019). Isolation, identification and characterization of endophytic bacteria antagonistic to Phytophthora palmivora causing black pod of cocoa in Malaysia. European Journal of Plant Pathology, 155, 1077-1091.

Berger, R.D. (1981). Comparison of the Gompertz and logistic functions to describe plant disease progress, Phytopathology, 71, 716-719.

Campbell, A.; R. Ploetz \& O. Gutierrez (2015). Using digital image analysis to evaluate disease resistance. $2^{\text {nd }}$ Annual Meeting of the Americas Cacao Breeders' Working Group, San Salvador.

Cilas, C. \& D. Despréaux (2004). Improvement of Cocoa Tree Resistance to Phytophthora Diseases. CIRAD, France.

Contreras-Medina, L.M.; I. Torres-Pacheco; R.G. Guevara-González; R.J. RomeroTroncoso; I.R. Terol-Villalobos \& R.A. Osornio-Rios (2009). Mathematical modeling tendencies in plant pathology. African Journal of Biotechnology, 8, 7399-7408.

Drenth, A. \& B. Sendall (2004). Isolation of Phytophthora from infected plant tissue and soil and principles of species identification. p. 94-102. In: Diversity and Management of Phytophthora in Southeast Asia, (A. Drenth \& D.I. Guest, Eds). ACIAR Monograph No. 114, Melbourne. 
Efombagn, M.I.B.; D. Bieysse; S. Nyasse \& A.B. Ekses (2011). Selection for resistance to Phytophthora pod rot of cocoa (Theobroma cacao L.) in Cameroon: Repeatability and reliability of screening tests and field observations. Crop Protection, 30, 105-110.

Forbes, G.; W. Pérez \& J.A. Piedra (2014). Field Assessment of Resistance in Potato to Phytophthora infestans. International Potato Center (CIP). Lima, Peru.

Haya, R.; M.Y. Nuraziawati; M.H. Jaafar \& M.M. Qahar (2012). Book of Malaysia Cocoa Clones. Malaysian Cocoa Board. Sabah, Malaysia.

Jain, A.K. (2010). Data clustering: 50 years beyond k-means. Pattern Recognition Letters, 31, 651-666.

Janani, P. \& N. Kumar (2014). Screening of cocoa genotypes against Phytophthora pod rot disease under hot tropical condition of Tamil Nadu. Trends in Biosciences, 7, 2484-2487.

Lee, C.H.; A.S.C. Ling; S.S. Soetikno; N.C.K. Jeremy; K. Ramle; S. Sabariah \& R. Haya (2014). Training Manual of Cocoasafe: Capacity Building and Knowledge Sharing in SPS in Cocoa in Southeast Asia (STDF/PG/381), Malaysian Cocoa Board, Kota Kinabalu, Sabah, Malaysia.

Lee, C.H.; L. Kelvin; R. Haya; M. Navies \& B. Saripah (2013). Cocoa Planting Manual, Sustainable Cocoa. Malaysian Cocoa Board, Kota Kinabalu, Sabah, Malaysia.

Ling, A.S.C.; A.K. Mohd Jaaffar; K.P. Chong \& C.M. Ho (2017). Assessing the cocoa genotypes for resistance to black pod using the area under the diseaseprogress curve (AUDPC). Bulgarian Journal of Agricultural Science, 23, 972-979.

Ling, A.S.C.; K.P. Chong \& C.M. Ho (2018). Comparing nonlinear models in describing disease progress curve of cocoa black pod. p. 040008(1-8) In: Proceedings of The $25^{\text {th }}$ National Symposium on Mathematical Sciences (SKSM25),
(M. Daud; A. Ajab Bai; M. Hamidah; M.J. Maheran; M. Mesliza; R.S. Sarifah \& W.A.R. Wan Eny Zarina, Eds.). Pahang.

Machiavelli, R.E. (2013). Non-linear mixed models for disease incidence and severity: Modeling plant diseases in tropical crops. p. 1057-1062. In: Proceedings of $59^{\text {th }}$ World Statistics Congress of the International Statistical Institute, Hong Kong.

Mau, Y.S. \& A.S.S. Ndiwa (2018). Field evaluation of late leaf spot and leaf rust resistance and the associated yield losses in Indonesian groundnut genotypes. Asian Journal of Plant Sciences, 17, 134-141.

Meena, P.D.; C. Chattopadhyay; S.S. Meena \& A. Kumar (2011). Area under disease progress curve and apparent infection rate of Alternaria blight disease of Indian mustard (Brassica juncea) at different plant age. Archives of Phytopathology and Plant Protection, 44, 684-693.

Mukherjee, A.K.; N.K. Mohapatra \& P. Nayak (2010). Estimation of area under the disease progress curves in a rice-blast pathosystem from two data points. European Journal of Plant Pathology, 127, 33-39.

Nayak, P.; A.K. Mukherjee; E. Pandit \& S.K. Pradhan (2018). Application of statistical tools for data analysis and interpretation in rice plant pathology. Rice Science, 25 , $1-18$.

Nyadanu, D.; M.K. Assuah; B. Adomako; Y.O. Asiama; I. Y. Opoku \& Y. AduAmpomah (2009). Efficacy of screening methods used in breeding for black pod disease resistance varieties in cocoa. African Crop Science Journal, 17, 175-186.

Nyadanu, D.; R. Akromah; B. Adomako; C. Kwoseh; S.T. Lowor, H. Dzahini-Obiatey, A.Y. Akrofi \& M.K. Assuah (2012). Inheritance and general combining ability studies of detached pod, leaf disc and natural field resistance to Phytophthora palmivora 
and Phytophthora megakarya in cacao (Theobroma cacao L.). Euphytica, 188, 253-264.

Ohunakin, A.O.; A.C. Odiyi \& B.O. Akinyele (2019). Comparison of rank sum and Area under Disease Progress Curve (AUDPC) as determinant for relative resistance status of maize populations to Northern leaf blight disease of maize. Advance in Plants \& Agriculture Research, 9, 395-400.

Pratama, S.W.; S. Sukamto; I.N. Asyiah \& Y.V.Ervina (2013). Growth inhibition of cocoa pod rot fungus Phytophthora palmivora by Pseudomonas fluorescence and Bacillus subtilis bacteria. Pelita Perkebunan, 29, 120-127.

SAS Institute (2013). SAS/STAT ${ }^{\circledR} 13.1$ User's Guide. SAS Institute Inc., Cary.

Sastry, K.S. \& T. A. Zitter (2014). Ecology and epidemiology of virus and viroid diseases of tropical crops. p. 1-148. In: Plant Virus and Viroid Diseases in the Tropics. Volume 2: Epidemiology and Management (K.S. Sastry \& T.A. Zitter, Eds), Springer, Dordrecht.
Simko, I. \& H. Piepho (2012). The area under the disease progress stairs: calculation, advantage, and application. Phytopathology, 102, 381-389.

Susilo, A.W. \& I. Anita-Sari (2014). Evaluation the resistance of cocoa clones (Theobroma cacao L.) to Phytophthora palmivora. Pelita Perkebunan, 30, 1-14.

Ten Hoopen, G.M.; P. Deberdt; M. Mbenoun \& C. Cilas (2012). Modelling cacao pod growth: implications for disease control. Annals of Applied Biology, 160, 260-272.

Tey, C.C. (1983). Black pod and brown pod of cocoa in Jerangau. MAPPS Newsletter, 7, 9-10.

Tondok, E.T.; M.S. Sinaga; Widodo \& M.T. Suhartono (2012). Potency of endophytic fungi as biocontrol agent of cacao black pod disease caused by Phytophthora palmivora (Butl.) Butl. Jurnal Agronomi Indonesia, 40, 146-152. 